\title{
金字塔频率特征融合目标检测网络
}

\author{
毛琳，李雪萌，，杨大伟，张汝波 \\ (大连民族大学机电工程学院 大连 116600) \\ (li_xmeng@126.com)
}

\begin{abstract}
摘 要: 针对深度学习网络在特征提取过程中运用上采样操作而致使细节纹理等高频特征缺失的问题, 提出一种金 字塔频率特征融合目标检测网络. 网络由 3 个深度学习金字塔网络构成, 输人图像经初级金字塔提取深度特征后, 分 别通过高频、低频增强金字塔形成不同的频率特征，利用特征融合来凸显深度学习网络在信息逐层传递过程中对细 节信息的保护能力，提高目标检测能力. 通过在分组角点检测网络(CornerNet)算法框架基础上仿真测试，该算法对 于目标模糊、目标重叠以及目标与背景反差小的情况，检测效果提升明显. 在 COCO 数据集上的检测结果与 CornerNet 算法相比, 平均精确率(average precision, AP)提高 $1 \%$ 以上, 尤其对行人、车辆等目标检测性能均有提高, 适用于无人驾驶系统与智能机器人等应用场景.
\end{abstract}

关键词：深度学习; 目标检测；金字塔网络；频率特征；特征融合

中图法分类号: TP391.41 DOI: 10.3724/SP.J.1089.2021.18306

\section{Pyramid Frequency Feature Fusion Object Detection Networks}

\author{
Mao Lin, Li Xuemeng ${ }^{*}$, Yang Dawei, and Zhang Rubo \\ (College of Mechanical and Electronic Engineering, Dalian Minzu University, Dalian 116600)
}

\begin{abstract}
For the problem of the absence of detail texture and other high-frequency features in the feature extraction process of deep learning network employing the up-sampling operation, a pyramid frequency feature fusion object detection network is proposed with three deep learning pyramid networks, to balance the high and low frequency feature information and improve the detection accuracy. The deep feature of the input image is extracted from the primary pyramid, different frequency characteristics are formed respectively by the high and low frequencies enhancement pyramid. In the process of information transmission, feature fusion is used to highlight the detail information protection ability of deep learning network and improve the object detection capability. After the simulation test based on CornerNet algorithm framework, the detection effect of proposed algorithm on vague objects, overlapping objects and low contrast between the objects and the background is significantly increased. The detection results on COCO dataset are more than $1 \%$ higher than CornerNet algorithm, so the proposed algorithm has a good performance in detecting pedestrians, vehicles and other objects, which is able to application autonomous vehicle systems and smart robots.
\end{abstract}

Key words: deep learning; object detection; pyramid networks; frequency characteristic; feature fusion

收稿日期：2020-04-08; 修回日期：2020-10-16. 基金项目：国家自然科学基金(61673084); 辽宁省自然科学基金(20170540192, 20180550866). 毛琳(1977一), 女, 博士, 副教授, 硕士生导师, 主要研究方向为多传感器信息融合、目标跟踪; 李雪萌(1997一), 女, 硕士研究生, 论文通讯作者, 主要研究方向为深度学习视觉处理技术; 杨大伟(1978一), 男, 博士, 副教授, 硕士生导师, 主要研究方 向为计算机视觉处理技术; 张汝波(1962一), 男, 博士, 副教授, 硕士生导师, 主要研究方向为智能机器人技术及智能信息处理技术. 
特征提取网络的结构设计是决定目标检测性 能高低的关键因素. 越深的网络意味着越强大的 特征提取能力, 为保证特征传递尺度不会过小, 某 些网络会在结构中加人上采样操作, 如典型的沙 漏网络(Hourglass) ${ }^{[1]}$. 这使得高频信息在传递过程 中出现损坏，从而打破特征的频率特性，细节信息 将得不到补偿, 直接影响检测器的性能评价.

使用特征提取网络的目标检测算法分为单阶 段和两阶段. 以区域卷积神经网络(regions with CNN features, RCNN $)^{[2-4]}$ 为代表的两阶段目标检测模型, 在卷积神经网络主干外辅助目标区域候选机制实现 目标分类功能. 单阶段目标检测包括 $\mathrm{YOLO}^{[5]}$ 、单次 多尺度检测器 (single shot multibox detector, SSD $)^{[6]}$, CornerNet 类型算法 ${ }^{[7-9]}$, 算法主要关注特征之间的 联系, 不需要候选区域来提供目标框的预测.

主流的深度学习目标检测算法大都使用 VGG-16, GoogLeNet, 残差网络 (residual neural networks, ResNet $)^{[10]}$ 等作为特征提取网络, 这些网 络的作用是提取图像中的特征信息用于后续的融 合、分类和回归等操作，仅通过单层特征进行处理, 对图像中的细节信息无法进行高精确率检测. 特 征金字塔网络(feature pyramid networks, FPN) ${ }^{[11]}$ 是 根据特征金字塔概念设计的特征提取器, 逐层传 递多尺度特征信息, 但是随着卷积层数增多, 特征 逐渐抽象，会减弱部分细节信息. Hourglass 使用 ResNet 作为初级特征提取模块, 经过一系列上采 样和融合操作提取多尺度特征, 能够保留输人图 像尺寸和整体特征信息, 但会损失一部分边缘纹 理特征, 尤其是目标与背景反差较小时, 检测失 效. 张思宇等 ${ }^{[12]}$ 结合 FPN 与 Hourglass 结构的特点, 在 SSD 网络中使用反卷积提升特征分辨率尺寸, 但过多的反卷积操作会增加计算量, 降低检测效 率. 张冬明等 ${ }^{[13]}$ 提出一种深度特征融合模型, 通 过融合低层显著特征图优化全卷积神经网络; 虽 然低层特征图包含更多的纹理信息, 但经过深度 提取易削弱细节特征的表达能力, 对于运动模糊 目标、部分遮挡目标以及目标与背景低反差时, 难 以精确地定位目标边界, 影响检测效果, 存在错检 或漏检现象.

为充分提取特征边缘细节信息，增强频率特 征的表达能力, 针对 Hourglass 结构的缺陷, 弥补 网络因深度提取缺失的高频信息, 本文提出金字 塔频率特征融合目标检测网络 (tri-pyramid frequency feature fusion object detection networks, TP-Net). 其采用 3 个特征金字塔结构，对特征进
行逐步提取，运用图像频率域高低频信息融合的 思想, 利用高低频 FPN 处理输人的频率信息, 改 善 Hourglass 因上采样处理而缺失细节纹理特征的 现象, 输出增强后的高低频率特征融合信息, 提高 特征传递的稳定性, 有效地降低目标模糊、目标重 叠以及目标与背景低反差引起的漏检或错检概率.

\section{1 金字塔频率特征融合网络}

在卷积神经网络中, 原始图像由高频信息和 低频信息共同构成, 高频图像信息边缘细节明显, 低频图像信息内部特征完整. 低频信息由网络经 过逐层上采样得到低频截止频率, 从而获得稳定 的低频特征信息. 随着网络不断迭代训练, 通过卷 积计算促使损失值下降, 最后趋于稳定, 在网络进 行反向传播过程中不断弥补因提取低频信息而缺 失的高频信息. 通过分离特征高低频率信息, 充分 提取相关特征信息并进行有效融合, 可以增强特 征表达能力. 如图 1 所示, TP-Net 分为 3 级结构, I 级由初级特征金字塔 $P_{1}$ 构成, 采用残差模块作 为初级特征提取模块, 逐步提取深度特征; 在 II 级 中, 针对特征不同频率, 将网络分为低频增强金字 塔 $P_{2}$ 和高频增强金字塔 $P_{3}$ 进行频率提取; 在III级 中, 根据高低频增强信息进行自适应加权融合, 输 出特征结果. 与 Hourglass 相比, TP-Net 弥补了 Hourglass 在特征传递过程中缺失的纹理信息, 在 保证计算效率的前提下既能将高频特征中几何表 征能力强的优势保留, 又能融合有效的低频特征, 进一步完善特征信息, 保持特征传递的稳定性, 有 效地提升预测图像的质量.

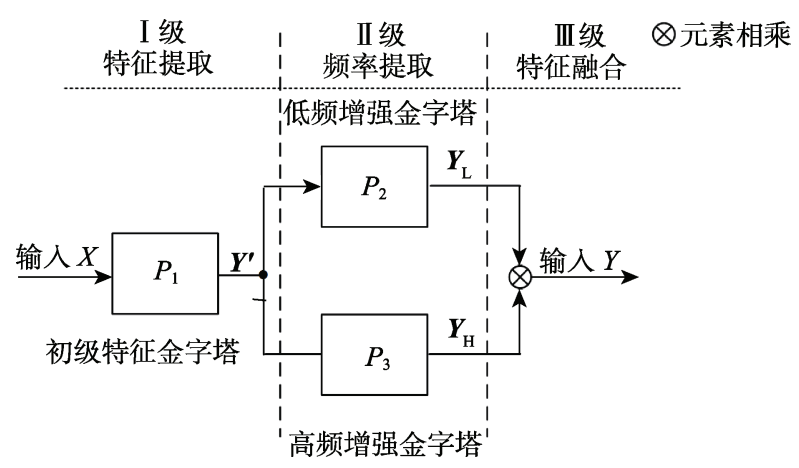

图 1 TP-Net 流程图

\section{1 初级特征金字塔}

特征提取网络由初级特征金字塔构成，其功 能是对输人图像进行深度特征提取. TP-Net在特征 提取过程中采用与 Hourglass 相近的结构, 具有多 
尺度信息获取检测能力.

TP-Net 初级特征提取模块内部有 2 条支路, 采用残差金字塔结构，通过快捷连接线返回原尺 度特征, 经一系列上采样输出多尺度信息. 因采用 ResNet 作为初级特征提取模块, 能够保留原有特 征的层次信息, 还能保持输入输出尺寸不变.

定义 1. 残差模块的数学表达为

$$
\begin{gathered}
\boldsymbol{F}_{t}(x)=\boldsymbol{W}_{2} * \sigma\left(\boldsymbol{W}_{1} * \boldsymbol{X}_{l-1}\right) \\
\boldsymbol{Y}^{\prime}=\boldsymbol{F}_{t}\left(\boldsymbol{X}_{l-1}\right)+\boldsymbol{X}_{l-1}
\end{gathered}
$$

其中, $\boldsymbol{X}, \boldsymbol{Y}^{\prime} \in \mathbb{R}^{c \times h \times w}$ 分别为输人输出特征张量, $h$ 和 $w$ 表示空间维度, $c$ 表示通道数; $\boldsymbol{F}_{t}(x)$ 作为初级 特征金字塔中若干堆叠层拟合的基础映射, $l \in \mathbb{Z}^{+}$ 表示特征层; $\sigma($.$) 为 ReLU 激活函数; \boldsymbol{W}_{1}$ 和 $\boldsymbol{W}_{2}$ 是 权重矩阵.

利用映射得到的特征 $\boldsymbol{F}_{t}(x)$ 与原始特征 $\boldsymbol{X}$ 进行 像素点的叠加融合, 能够缓解梯度消失, 提高特征 传递效率。

\section{2 低频增强金字塔}

频率融合网络由高频低频增强金字塔网络构 成. 低频增强金字塔的功能是对图像的低频特征 进行增强处理. 图像经初级特征金字塔输出的融 合信息进人低频增强金字塔，经过多层最近邻域 上采样操作. 该方法以最近的已知像素的灰度值 作为所需插值像素点的灰度值. 考虑了所需插值 像素点与其相邻像素点之间多数情况下相关性较 大的事实，能在一定程度上保持图像清晰度; 但其 针对边缘细节信息表达不明显，导致高频信息被 过滤. 因此, 根据采样定理可知, 上采样操作相当 于对特征插值计算后低通滤波，即低频增强金字 塔等效为低通增强滤波器 ${ }^{[14]}$

在低频处理的同时，与残差快捷连接线返回 的多尺度特征叠加融合，在保持特征尺寸不发生 变化的情况下，针对低频信息不断提取并融合，致 使低频信息得到有效提取，增强低频信息.

定义 2. 低频增强金字塔的数学表达为

$$
\begin{aligned}
& \hat{\boldsymbol{X}}=\boldsymbol{F}_{\mathrm{L}}\left[\boldsymbol{F}_{\mathrm{L}}\left(\boldsymbol{X}_{l-1}\right)+\boldsymbol{X}_{l-1}\right] \\
& \boldsymbol{Y}_{\mathrm{L}}=\frac{1}{N}\left\{\boldsymbol{U}[\boldsymbol{U}(\hat{\boldsymbol{X}})]+\boldsymbol{X}_{l-1}\right\}
\end{aligned}
$$

其中, $\boldsymbol{X} \in \mathbb{R}^{c \times h \times w}$ 为输入特征张量, $\boldsymbol{Y}_{L} \in \mathbb{R}^{c \times h \times w}$ 为 低频增强金字塔输出的低频增强分量, $h$ 和 $w$ 表示 空间维度, $c$ 表示通道数; $\boldsymbol{F}_{\mathrm{L}}(x)$ 作为低频增强金 字塔中若干堆叠层拟合的基础映射. $\boldsymbol{X}_{l-1} \in$ $\left[x_{0}, x_{1}, \cdots, x_{l-1}\right]$ 是 $0, \cdots, l-1$ 层中产生的特征映射, $l \in \mathbb{Z}^{+}$表示特征层; $\boldsymbol{U}$ 为最近邻域上采样函数; $\hat{\boldsymbol{X}}$ 为低频增强金字塔拟合矩阵得到的融合估计值; $N \in \mathbb{R}^{c \times h \times w}$ 为归一化参数.

\section{3 高频增强金字塔}

与低频增强金字塔相对应，高频增强金字塔 的功能是对图像的高频特征进行增强处理. 高频 信息的缺失会使特征边缘细节信息不清晰，对于 目标模糊、目标重叠以及目标与背景反差小的场景 容易丧失检测能力.

为保证高频信息的完整准确获得，与低频处 理不同, 仅需要从初级特征金字塔中通过快捷连 接(shortcut)的方式提取已经抽象完成的高频频率 特征即可. 采用反卷积操作, 通过叠加卷积层增加 卷积操作, 对高频信息进行不断提取, 获得更多的 特征信息, 极大程度上保留特征细节信息, 弥补缺 失的特征信息，增强高频信息; 通过对网络结构的 加深和加宽, 保证频率信息稳定、有效地传递和减 少信息丢失.

定义 3. 高频增强金字塔的数学表达为

$$
\begin{gathered}
\hat{\boldsymbol{x}}=\boldsymbol{F}_{\mathrm{H}}\left[\boldsymbol{F}_{\mathrm{H}}\left(\boldsymbol{X}_{l-1}\right)+\boldsymbol{X}_{l-1}\right] \\
\boldsymbol{Y}_{\mathrm{H}}=\frac{1}{N}\left\{\boldsymbol{W}_{1} * \sigma[\boldsymbol{D}(\hat{\boldsymbol{x}})]+\boldsymbol{W}_{1} * \hat{\boldsymbol{x}}\right\}
\end{gathered}
$$

其中, $\boldsymbol{X} \in \mathbb{R}^{c \times h \times w}$ 为输人特征张量, $\boldsymbol{Y}_{\mathrm{H}} \in \mathbb{R}^{c \times h \times w}$ 为 输出高频特征张量, $h$ 和 $w$ 表示空间维度, $c$ 表示通 道数; $\boldsymbol{F}_{\mathrm{H}}(x)$ 作为高频增强金字塔中若干堆叠层 拟合的基础映射， $\hat{\boldsymbol{x}}$ 为 TP-Net 拟合矩阵的估计值; $\boldsymbol{D}(x)$ 为反卷积函数; $N \in \mathbb{R}$ 为归一化参数. $\boldsymbol{X}_{l-1} \in\left[x_{0}, x_{1}, \cdots, x_{l-1}\right]$ 是指 $0, \cdots, l-1$ 层中产生的特 征映射, $l \in \mathbb{Z}^{+}$表示特征层; $\sigma(\cdot)$ 为 ReLU 激活函 数; $\boldsymbol{W}_{1}$ 和 $\boldsymbol{W}_{2}$ 是权重矩阵.

\section{4 特征融合}

特征经高频低频增强金字塔，将低频增强金 字塔得到的低频信息与高频增强金字塔增强的高 频信息融合, 计算当前像素点与特征图内所有像 素点间的相似性估计值，通过空间全局信息增强 得到融合高低频信息的目标特征 $\boldsymbol{Y}$.

定义 4. 高低频融合张量的计算过程为

$$
\boldsymbol{Y}=\alpha \boldsymbol{Y}_{\mathrm{H}}^{p, q}+\beta \boldsymbol{Y}_{\mathrm{L}}^{p, q}
$$

$$
\begin{array}{r}
\boldsymbol{Y}=\alpha \sum_{j \in N_{k}}^{i \in N_{k}} \boldsymbol{W}_{i+\frac{k-1}{2}, j+\frac{k-1}{2}}^{\mathrm{T}} \boldsymbol{Y}_{\mathrm{H}}^{(p+i),(q+j)}+ \\
\beta \sum_{j \in N_{k}}^{i \in N_{k}} \boldsymbol{W}_{i+\frac{k-1}{2}, j+\frac{k-1}{2}}^{\mathrm{T}} \boldsymbol{Y}_{\mathrm{L}}^{\left(\left[\frac{p}{2}\right]+i\right),\left(\left[\frac{q}{2}\right]+j\right)}
\end{array}
$$


其中, $\boldsymbol{Y}_{\mathrm{H}}^{p, q}, \boldsymbol{Y}_{\mathrm{L}}^{p, q} \in \mathbb{R}^{c \times h \times w}, h$ 和 $w$ 表示空间维度, $c$ 表 示通道数; $p, q \in \mathbb{R}$ 是 $\boldsymbol{Y}_{\mathrm{H}}, \boldsymbol{Y}_{\mathrm{L}}$ 高低频张量中的位置坐 标. $\boldsymbol{Y}_{\mathrm{H}}^{p, q}$ 和 $\boldsymbol{Y}_{\mathrm{L}}^{p, q}$ 分别表示 $p, q$ 位置的高、低频率特征 张 量. $i=\left[-\frac{k-1}{2}, \cdots, \frac{k-1}{2}\right], j=\left[-\frac{k-1}{2}, \cdots, \frac{k-1}{2}\right]$, $(k \in 0,1,2, \cdots)$ 定义了一个局部区域， $(i, j)$ 表示所取 的近邻范围, $N_{k}=(i, j) ; \alpha, \beta \in[0,1]$ 分别为高频 融合系数和低频融合系数.

为维持融合后的频率特征稳定性, 需合理计 算相应系数; 频率特征的能量值大小决定了不同 频率特征融合系数的取值.

定义 5. 特征频率谱的数学表达为

$$
\begin{aligned}
& P(u, v)=|F(u, v)|^{2}=|F[f(x, y)]|^{2}= \\
& \left|\frac{1}{M N} \sum_{x=0}^{M-1} \sum_{y=0}^{N-1} f(x, y) \exp \left[-\mathrm{j} 2 \pi\left(\frac{x u}{M}+\frac{y v}{N}\right)\right]\right|^{2}
\end{aligned}
$$

频率特征能量谱的数学表达为

$$
S(\boldsymbol{Y})=\sum_{u}^{x} \sum_{v}^{y}|\boldsymbol{F}(\boldsymbol{Y})|^{2}
$$

其中, $x \in[0,1, \cdots, M-1], y \in[0,1, \cdots, N-1]$ 是输人 实变量; $u \in[0,1, \cdots, M-1], v \in[0,1, \cdots, N-1]$ 是输 人频率变量; $M, N$ 为特征空间维度; $P$ 为输人频率 特征的频率谱; $f(x, y)$ 为输人图像的原始映射函 数; $F(u, v)$ 为输人频率特征的映射函数; $\boldsymbol{F}$ 为傅里
叶变换; $S$ 为频率特征能量谱.

根据频率特征能量值大小，确定频率融合系 数取值, 其数学表达为

$$
\begin{aligned}
& \alpha=\frac{A}{\sqrt{2 \pi}} \exp \left\{-\frac{\left[S\left(\boldsymbol{Y}_{\mathrm{H}}\right)-0.1\right]^{2}}{2}\right\} \\
& \beta=\frac{B}{\sqrt{2 \pi}} \exp \left\{-\frac{\left[S\left(\boldsymbol{Y}_{\mathrm{L}}\right)-0.1\right]^{2}}{2}\right\}
\end{aligned}
$$

其中, $A, B \in[0,1]$ 为缩放系数; $\boldsymbol{Y}_{\mathrm{H}}=f_{\mathrm{H}}(x, y)$, $\boldsymbol{Y}_{\mathrm{L}}=f_{\mathrm{L}}(x, y)$.

因为 TP-Net 结合了残差模块的输出和 Hourglass 的输出, 弥补网络因上采样而缺失的细 节信息，极大程度保留了高频图像特征，通过融合 能够输出更多的边缘细节信息, 增强空间维度特 征表达能力，提高频率特征传递的稳定性.

\subsection{TP-Net 结构}

TP-Net 采用 3 个不同功能的金字塔 $P_{1}, P_{2}$ 和 $P_{3}$ 构成网络主体结构. 初级特征金字塔 $P_{1}$ 完成对输 人图像的逐步提取，随后将得到的深度特征分成 高低频两路, 一路输送到低频增强金字塔 $P_{2}$ 中, 逐层处理低频信息, 增强低频特征; 另一路输送到 高频增强金字塔 $P_{3}$ 中, 提取细节信息, 增强高频 特征. 将两路输出融合, 输出稳定的高低频率特征 融合信息 $\boldsymbol{Y}$. TP-Net 结构如图 2 所示.

$\oplus$ 元素相加

$\otimes$ 元素相乘

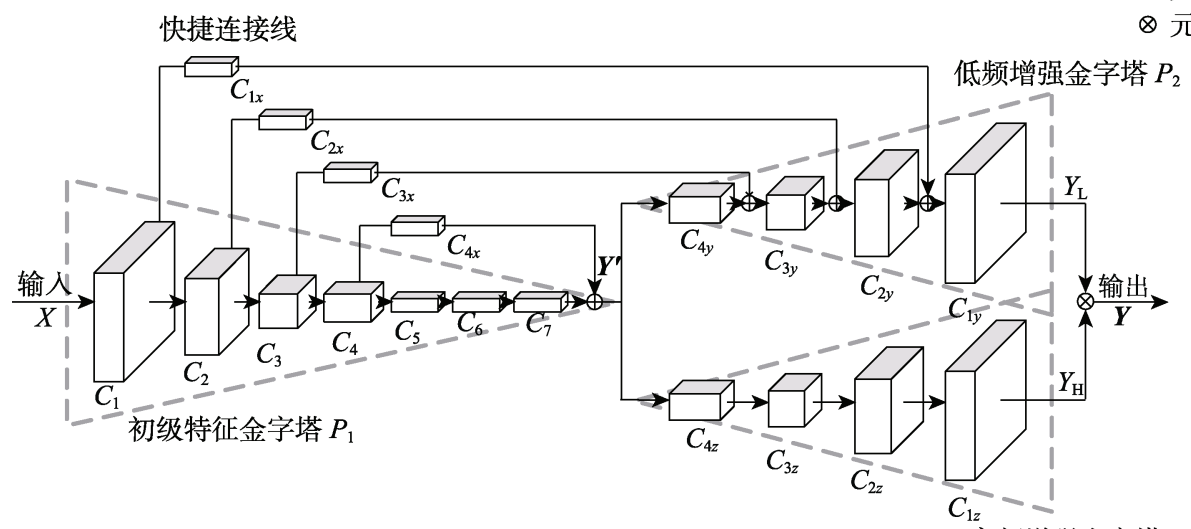

图 2 TP-Net 结构

TP-Net 特征提取流程如下.

Step1. 将图像输人到初级特征金字塔 $P_{1}$ 中, 逐层 提取主要特征. 首先由 $C_{1}$ 层逐层进行最大池化操作, 对 特征信息进行降维，避免发生过拟合，同时对特征进行 压缩.

Step2. 特征从 $C_{1}$ 到 $C_{7}$ 层完成基本提取，与残差快 捷连接线进行融合，此时输出融合特征.
Step3. 在低频增强金字塔 $P_{2}$ 中, 经过 $C_{4 y}$ 到 $C_{1 y}$ 逐 层进行上采样, 并与 $C_{4 x}$ 到 $C_{1 x}$ 残差快捷连接线返回的 特征融合, 返回特征至输人特征尺度的特征映射.

Step4. 特征经 $C_{7}$ 与 $C_{4 x}$ 融合后提取能力最强, 在 高频增强金字塔 $P_{3}$ 中, 将融合得到的深度特征及时提 取出来, 先经 $C_{4 z}$ 反卷积再通过 3 个卷积层使高低频输 出的特征图具有相同的维度特性，保留高频信息，消除 
上采样带来的混叠效应, 及时补足因高频信息缺失所减 少的边缘细节特征.

Step5，直接将低频增强金字塔 $P_{2}$ 与高频增强金字 塔 $P_{3}$ 的输出叠加, 融合低频信息同时增强高频信息, 输 出高低频率融合的特征张量 $\boldsymbol{Y}$, 提升目标边缘细节特征 的表达能力.

经 TP-Net 增强得到的特征与 Hourglass 输出的 特征可视化效果对比如图 3 所示.

通过图 3e 和图 3i 可视化输出对比可知, 经过高 频增强后, 自行车轮廓细节突出明显; 通过图 3f 和 图 $3 \mathrm{j}$ 可视化输出对比可知, 斑马条纹及轮廓信息得 到增强. 通过增强边缘特征, 图 $3 \mathrm{~g}$ 和图 $3 \mathrm{k}$ 可视化输
出对比中位置交错的 5 个人以及图 $3 \mathrm{~h}$ 和图 31 对比中 2 只长颈鹿均得以有效区分, 其与背景的反差效果也 明显提升. 通过对比 Hourglass 与 TP-Net 的输出特 征, 可知特征高频信息对于增强目标的边缘细节特 征效果明显; 图 3m 图 3p 都是 TP-Net 与 Hourglass 的输出特征频率曲线, 在网络训练过程中, 提取低 频增强金字塔输出层特征即原始 Hourglass 输出层特 征 $C_{1 y}$ 与高频增强金字塔输出层特征 $C_{1 z}$, 计算所提 取的全部特征的频率平均值进行对比分析. 曲线中 TP-Net 高频信息相比 Hourglass 提升显著，在一定程 度上提高了特征的表达能力, 利于目标的检测.

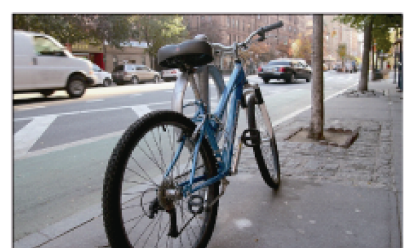

a. 原图 1

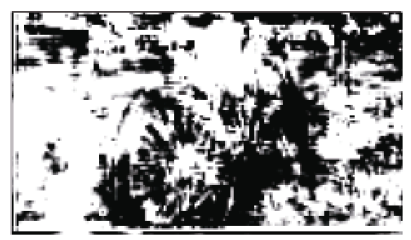

e. Hourglass 输出 1

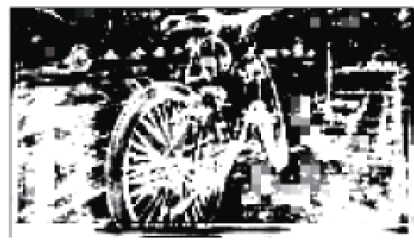

i. TP-Net 输出 1

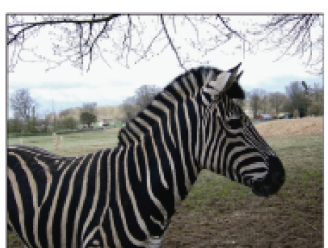

b. 原图 2

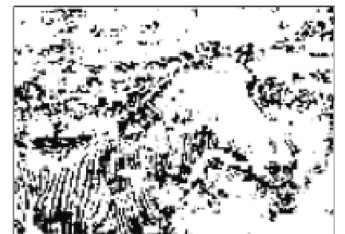

f. Hourglass 输出 2

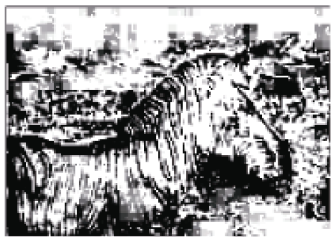

j. TP-Net 输出 2

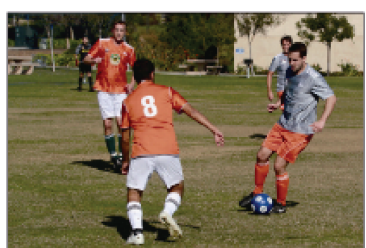

c. 原图 3

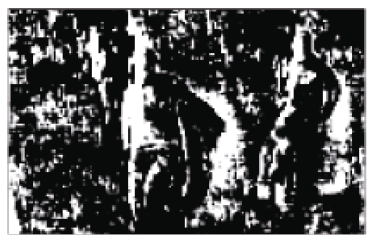

g. Hourglass 输出 3

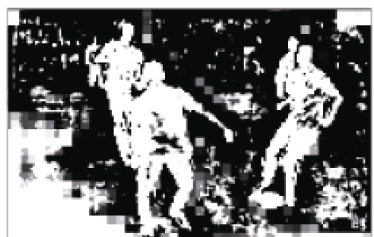

k. TP-Net 输出 3

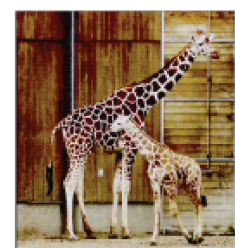

d. 原图 4

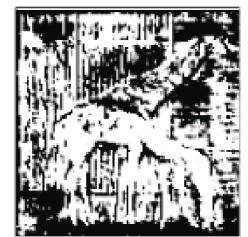

h. Hourglass 输出 4

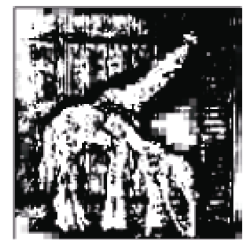

1. TP-Net 输出 4
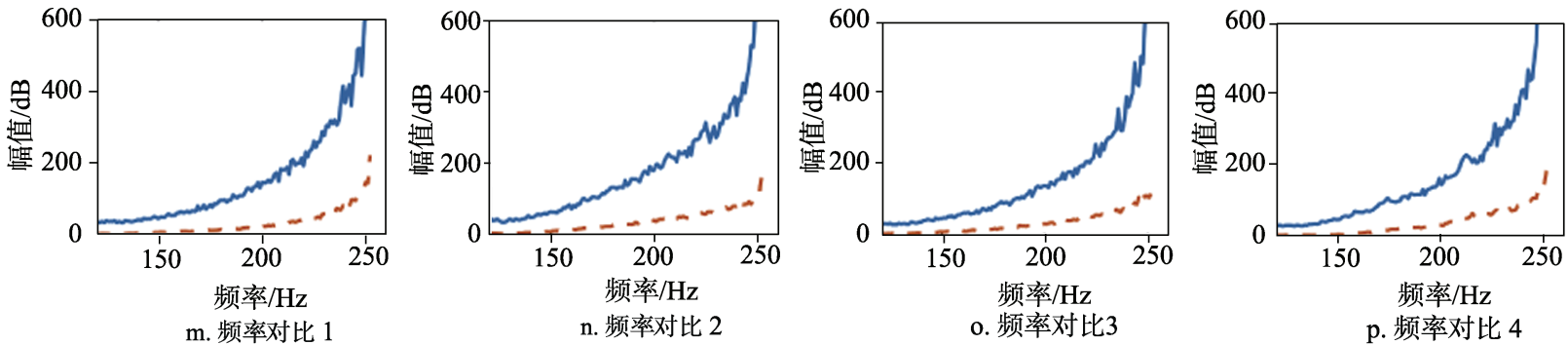

$\longrightarrow$ Hourglass $^{[1]} ; \ldots$...- TP-Net

图 3 不同网络特征可视化对比

\section{2 实验及结果分析}

\section{1 实施细节设置}

实验基于 CornerNet 目标检测算法框架，以 TP-Net 为骨干网络替代 Hourglass 网络. 运行环境 硬件配置为 8 张 NVDIA 1080Ti 显卡, 软件系统为
Ubuntu 16.04, 实验程序在 Pytorch 0.4.0 深度学习框 架下使用 Python 编程语言实现. 算法超参数 batch size 设置为 28 , 学习率为 0.00005 , 迭代次数为 150000 . 使用通用场景目标 (common objects in context, COCO $)^{[15]} 2014$ 数据集, 训练集共有 123287 幅图像, 包含 80 个类别. COCO 使用不同交并比 
(intersection over union, IoU) 和平均精确率 (average precision, AP)、平均召回率(average recall, AR)评估检测器性能. 其中, AP 是在 10 个不 同的 IoU 阈值 $(0.50 / 0.05 / 0.95)$ 和所有类别上计算 的, 被认为是 MSCOCO 数据集上最重要的指标. $\mathrm{AR}$ 是在每幅图像的固定数量(即 1,10 和 100)的检 测上计算的，并在所有类别和 10 个不同的 IoU 阈 值上进行平均. 此外, AP 和 AR 可用于评估不同目
标比例下的性能, 包括小目标 $($ 面积 $<32 \times 32)$ 、中等 目标 $(32 \times 32<$ 面积 $<96 \times 96)$ 和大目标 $($ 面积 $>96 \times 96)$.

\section{2 算法对比}

为验证 TP-Net 对主体网络框架检测的提升效 果，本文基于 CornerNet 目标检测算法，以 TP-Net 作为骨干网络, 与使用 Hourglass 网络作为骨干网 络的原始 CornerNet 算法以及其他目标检测算法进 行对比，结果如表 1 所示.

表 1 目标检测算法对比

\begin{tabular}{|c|c|c|c|c|c|c|c|c|c|c|c|c|c|c|}
\hline & 方法 & 骨干网络 & $\mathrm{AP}$ & $\mathrm{AP}^{50}$ & $\mathrm{AP}^{75}$ & $\mathrm{AP}^{\mathrm{s}}$ & $\mathrm{AP}^{\mathrm{m}}$ & $\mathrm{AP}^{1}$ & $\mathrm{AR}^{1}$ & $\mathrm{AR}^{10}$ & $\mathrm{AR}^{100}$ & $\mathrm{AR}^{\mathrm{s}}$ & $\mathrm{AR}^{\mathrm{m}}$ & $\mathrm{AR}^{1}$ \\
\hline \multirow{4}{*}{ 两阶段 } & Faster-RCNN & Inception-ResNet-v $2^{[16]}$ & 34.7 & 55.5 & 36.7 & 13.5 & 38.1 & 52.0 & \multirow{4}{*}{21.7} & \multirow{4}{*}{43.7} & \multirow{4}{*}{50.9} & & & \\
\hline & $\mathrm{D}-\mathrm{FCN}^{[17]}$ & Aligned-Inception-ResNet & 37.5 & 58.0 & & 19.4 & 40.1 & 52.5 & & & & & & \\
\hline & Regionlets $^{[18]}$ & ResNet-101 & 39.3 & 59.8 & & 39.3 & 59.8 & & & & & & & \\
\hline & Mask-RCNN ${ }^{[19]}$ & ReNeXt-101 ${ }^{[20]}$ & 39.8 & 62.8 & 43.4 & 22.1 & 43.2 & 51.2 & & & & & & \\
\hline \multirow{6}{*}{ 一阶段 } & YOLOv2 $2^{[21]}$ & DarkNet-19 & 21.6 & 44.0 & 19.2 & 5.0 & 22.4 & 35.5 & 20.7 & 31.6 & 33.3 & 9.8 & 36.5 & 54.4 \\
\hline & $\mathrm{SSD}^{[6]}$ & ResNet-101 & 31.2 & 50.4 & 33.3 & 10.2 & 34.5 & 49.8 & 28.3 & 42.1 & 44.4 & 17.6 & 49.2 & 65.8 \\
\hline & $\operatorname{DSSD}^{[22]}$ & ResNet-101 & 33.2 & 53.3 & 35.2 & 13.0 & 35.4 & 51.1 & \multirow[t]{2}{*}{28.9} & \multirow[t]{2}{*}{43.5} & \multirow[t]{2}{*}{46.2} & \multirow[t]{2}{*}{21.8} & \multirow[t]{2}{*}{49.1} & \multirow[t]{2}{*}{66.4} \\
\hline & RefineDet $^{[23]}$ & ResNet-101 & 36.4 & 57.5 & 39.5 & 16.6 & 39.9 & 51.4 & & & & & & \\
\hline & CornerNet & Hourglass-104 & 39.1 & 54.6 & 41.6 & 18.7 & 41.8 & 54.2 & 34.7 & 53.4 & 58.1 & 37.4 & 61.5 & 75.6 \\
\hline & CornerNet & TP-Net & 40.2 & 55.8 & 42.8 & 19.7 & 42.9 & 55.9 & 35.0 & 54.1 & 58.9 & 38.9 & 62.5 & 76.8 \\
\hline
\end{tabular}

注. 加粗文字为本文算法结果; 上标 50,75 分别代表 IOU 取值为 0.50 和 $0.75 ; \mathrm{s}, \mathrm{m}, 1$ 分别代表小、中等和大目标; $1,10,100$ 代 表每幅图像的固定数量.

增强高频信息后的 TP-Net 相较于其他主干目 标检测网络的检测精确率有明显提升, 实现了 $40.2 \%$ 的 AP, 结果显示本文算法在各项指标中不仅 比 YOLO, SSD 等一阶段目标检测算法检测效果提 升显著, 并且能够与 Mask-RCNN 等两阶段目标检 测算法相謧美. 结果表明，在目标检测算法中，图 像频率传递不平衡对检测精确率影响显著, 实验验 证了对图像进行高低频率特征融合处理的可行性.

测试结果显示, 使用 TP-Net 作为骨干网络比 使用 Hourglass 作为骨干网络原始的 CornerNet 目 标检测算法 AP 提高 $1 \%$ 以上. 在 COCO2014 数据 集 80 个检测类别中，采用 TP-Net 的 CornerNet 检 测算法的平均精确率均优于原始网络, 表 2 所示为 18 个类别的对比结果.

采用 TP-Net 作为主干网络的目标检测算法对 人、交通工具、动物等类别目标的检测效果提高尤 其明显. 当应用于无人驾驶系统、智能机器人等领 域时, 能够有效提高检测精确率, 相比原始 Hourglass 网络具有较大优势. 结果表明在目标检 测算法中，图像频率传递不平衡对检测精确率影 响显著, 实验验证了对图像进行高低频率特征融 合处理的可行性.

\section{3 消融实验}

经过与不同方法的对比实验，已验证本文方
法的有效性和可行性. 为了进一步验证 TP-Net 骨 干网络的泛化性, 本节通过消融实验, 验证 TP-Net 在目标检测算法中的有效性. 对作用于同 - CornerNet 目标检测算法, 表 3 所示为使用不同 骨干网络对其影响.

表 2 在 COCO2014 数据集上的仿真结果 $\quad \%$

\begin{tabular}{lcc}
\hline \multicolumn{1}{c}{ 类别 } & Hourglass $^{[1]}$ & TP-Net \\
\hline person & 45.8 & 47.0 \\
bicycle & 29.4 & 31.3 \\
car & 38.3 & 39.4 \\
bus & 64.5 & 65.7 \\
train & 65.1 & 66.2 \\
truck & 36.5 & 38.6 \\
stop sign & 63.7 & 64.0 \\
suitcase & 34.8 & 36.6 \\
bench & 26.6 & 26.7 \\
cat & 68.3 & 71.2 \\
umbrella & 32.5 & 33.1 \\
handbag & 17.5 & 17.7 \\
traffic light & 21.4 & 21.8 \\
airplane & 65.0 & 66.7 \\
cell phone & 35.8 & 39.4 \\
boat & 19.4 & 21.7 \\
bird & 30.0 & 30.7 \\
dog & 64.4 & 65.9 \\
\hline
\end{tabular}


表 3 不同骨干网络检测对比

\begin{tabular}{lcccccc}
\hline \multicolumn{1}{c}{ 骨干网络 } & $\mathrm{AP}$ & $\mathrm{AP}^{50}$ & $\mathrm{AP}^{75}$ & $\mathrm{AP}^{\mathrm{s}}$ & $\mathrm{AP}^{\mathrm{m}}$ & $\mathrm{AP}^{\mathrm{1}}$ \\
\hline FPN $^{[1]]}$ & 30.2 & 44.1 & 32.0 & 13.3 & 33.3 & 42.7 \\
Hourglass $^{[1]}$ & 39.1 & 54.6 & 41.6 & 18.7 & 41.8 & 54.2 \\
TP-Net & $\mathbf{4 0 . 2}$ & $\mathbf{5 5 . 8}$ & $\mathbf{4 2 . 8}$ & $\mathbf{1 9 . 7}$ & $\mathbf{4 2 . 9}$ & $\mathbf{5 5 . 9}$ \\
\hline
\end{tabular}

注. 加粗文字为本文算法结果; 上标 50,75 分别代表 IOU 取值为 0.5 和 $0.75 ; \mathrm{s}, \mathrm{m}, 1$ 分别代表小、中等和大目标.

表 3 显示 CornerNet 使用 3 种骨干网络检测精 确率对比, TP-Net 对比 Hourglass 和 FPN 的 AP 分 别提升约 1.1\%和 $10.0 \%$. 结果表明，骨干网络的选 择很重要, TP-Net 对 CornerNet 的性能至关重要。

表 4 所示为不同检测算法对相同的 TP-Net 骨 干网络的适用性对比，加粗文字为本文算法结果.

表 4 显示，使用 TP-Net 代替原始骨干网络后，
CenterNet 算法的 AP 提升了 $0.7 \%$. 结果验证了 TP-Net 对于不同目标检测算法效果提升明显, 具 有一定的泛化能力.

表 4 不同算法框架检测对比

\begin{tabular}{clcccccc}
\hline 算法 & 骨干网络 & $\mathrm{AP}$ & $\mathrm{AP}^{50}$ & $\mathrm{AP}^{75}$ & $\mathrm{AP}^{\mathrm{s}}$ & $\mathrm{AP}^{\mathrm{m}}$ & $\mathrm{AP}^{1}$ \\
\hline \multirow{2}{*}{ CornerNet } & Hourglass $^{[1]}$ & 39.1 & 54.6 & 41.6 & 18.7 & 41.8 & 54.2 \\
& & & & & & & \\
& TP-Net & $\mathbf{4 0 . 2}$ & $\mathbf{5 5 . 8}$ & $\mathbf{4 2 . 8}$ & $\mathbf{1 9 . 7}$ & $\mathbf{4 2 . 9}$ & $\mathbf{5 5 . 9}$ \\
\hline \multirow{2}{*}{ CenterNet $^{[9]}$} & Hourglass $^{[1]}$ & 41.8 & 58.8 & 45.0 & 25.3 & 45.7 & 54.6 \\
& & & & & & & \\
& TP-Net & $\mathbf{4 2 . 5}$ & $\mathbf{6 0 . 0}$ & $\mathbf{4 5 . 5}$ & $\mathbf{2 3 . 9}$ & $\mathbf{4 6 . 4}$ & $\mathbf{5 7 . 0}$ \\
\hline
\end{tabular}

注. 加粗文字为本文算法结果.

2.4 性能分析及对比

替换主干网络为 TP-Net 的 CornerNet 目标检测 算法与原 Hourglass 网络测试结果对比如图 4 所示.

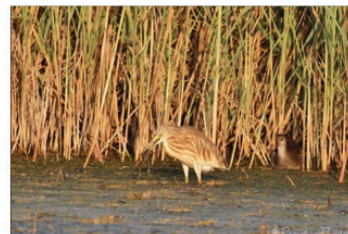

a. Hourglass 漏检 1

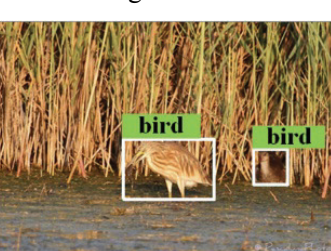

e. 本文正确检测 1

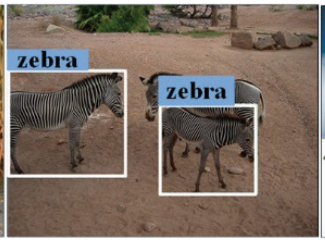

b. Hourglass 漏检 2

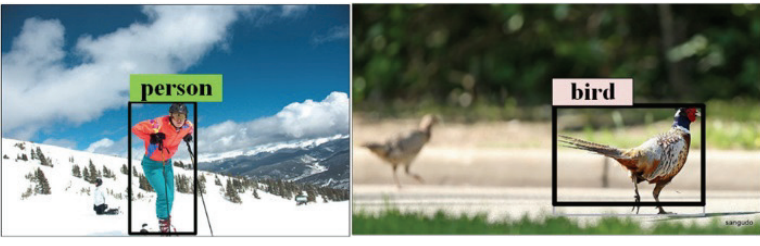

c. Hourglass 漏检 3

d. Hourglass 漏检 4

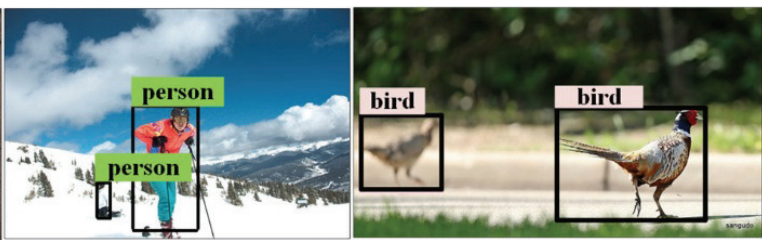

h. 本文正确检测 4

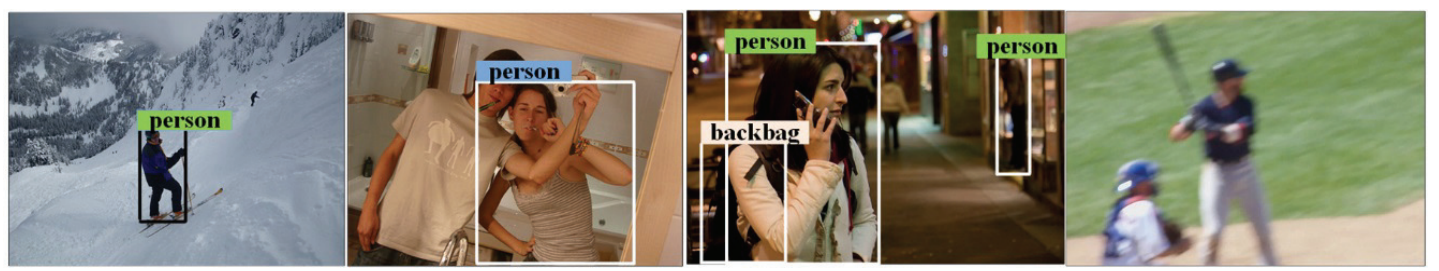

i. Hourglass 漏检 5

j. Hourglass 漏检 6

k. Hourglass 漏检 7

1. Hourglass 漏检 8

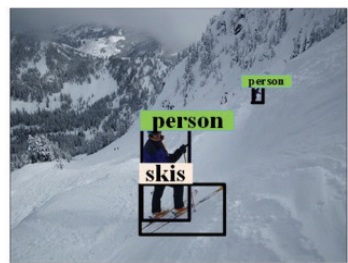

m. 本文正确检测 5

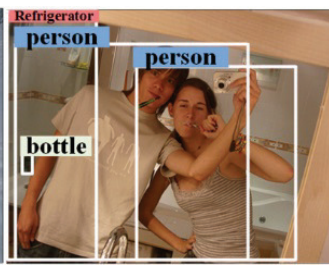

n. 本文正确检测 6

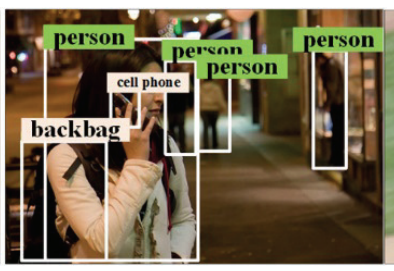

o. 本文正确检测 7

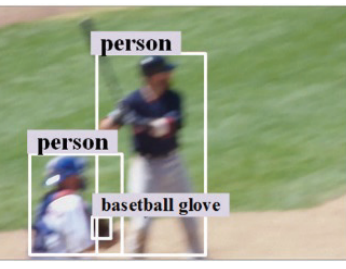

p. 本文正确检测 8

图 4 Hourglass 与 TP-Net 检测结果对比

从图 4a 和图 4e 对比中可以看出, 由于目标与 背景区分度过低，目标(右侧的小鸟)没有被检测 到, 但通过 TP-Net 能够准确地检测出图中目标; 在图 $4 \mathrm{~b}$ 和图 $4 \mathrm{f}$ 组与图 $4 \mathrm{j}$ 和图 $4 \mathrm{n}$ 组对比中, 目标 存在遮挡现象, 原网络由于边缘细节特征定位不 准确, 不能有效地检测重叠场景中的目标, 而 TP-Net 能够精确地识别出被遮挡的目标; 在图 $4 \mathrm{c}$
和图 $4 \mathrm{~g}$ 与图 $4 \mathrm{i}$ 和图 $4 \mathrm{~m}$ 对比中, 由于目标过小且 与背景反差小, 原网络出现漏检的现象, 而 TP-Net 准确地识别出未检测到的目标(图 4g 中左 侧的人, 图 $4 \mathrm{~m}$ 中右上的人); 在图 $4 \mathrm{~d}$ 和图 $4 \mathrm{~h}$ 组与 图 41 和图 $4 \mathrm{p}$ 对比中, 由于目标处于运动状态, 出 现模糊现象, TP-Net 能够识别漏检的模糊目标(图 $4 \mathrm{~h}$ 左侧小鸟, 图 $4 \mathrm{p}$ 中人物). 
对比原始 CornerNet算法检测结果, 采用 TP-Net 作为骨干网络后，算法的检测精确率得到提升，检 测效果得到增强. 虽然增加了卷积操作, 但训练时 间并没有较大差距. 补足高频信息, 增强边缘细节, 能够检测到更多特征信息，对于目标模糊、目标重叠 以及目标与背景反差小的场景检测效果提升明显, 其适用于复杂场景，在检测精确率上有明显的优势.

\section{3 结 语}

本文针对卷积神经网络特征传递频率不平衡 等问题，提出 TP-Net 频率特征融合网络，通过高、 低频增强金字塔分别处理不同频率信息, 并进行有 效融合提高特征传递的稳定性，显著提升检测性能. 与 Hourglass 相比, 进一步增强了目标的边缘细节特 征, 并得到较优检测结果, 提高对目标模糊、目标重 叠以及目标与背景反差小的场景的检测精确率，适 用于自动驾驶和机器人视觉等领域. 未来工作中, 将进一步优化特征融合效率，提升检测精确率.

\section{参考文献(References):}

[1] Newell A, Yang K Y, Deng J. Stacked Hourglass networks for human pose estimation[C] //Proceedings of European Conference on Computer Vision. Heidelberg: Springer, 2016: 483-499

[2] Girshick R, Donahue J, Darrell T, et al. Rich feature hierarchies for accurate object detection and semantic segmentation[C] // Proceedings of the IEEE Conference on Computer Vision and Pattern Recognition. Los Alamitos: IEEE Computer Society Press, 2014: 580-587

[3] Girshick R. Fast R-CNN[C] //Proceedings of the IEEE International Conference on Computer Vision. Los Alamitos: IEEE Computer Society Press, 2015: 1440-1448

[4] Ren S Q, He K M, Girshick R, et al. Faster R-CNN: towards real-time object detection with region proposal networks[J]. IEEE Transactions on Pattern Analysis and Machine Intelligence, 2017, 39(6): 1137-1149

[5] Redmon J, Divvala S, Girshick R, et al. You only look once: unified, real-time object detection[C] //Proceedings of the IEEE Conference on Computer Vision and Pattern Recognition. Los Alamitos: IEEE Computer Society Press, 2016: 779-788

[6] Liu W, Anguelov D, Erhan D, et al. SSD: single shot multibox detector[C] //Proceedings of the European Conference on Computer Vision. Heidelberg: Springer, 2016: 21-37

[7] Law H, Deng J. CornerNet: detecting objects as paired key-points [J]. International Journal of Computer Vision, 2018: 765-781

[8] Law H, Teng Y, Russakovsky O, et al. CornerNet-lite: efficient keypoint based object detection[OL]. [2020-04-08]. https: arxiv.org/abs/1904.08900

[9] Duan K W, Bai S, Xie L X, et al. CenterNet: object detection with keypoint triplets[C] //Proceedings of the IEEE International Conference on Computer Vision. Los Alamitos: IEEE
Computer Society Press, 2019: 6569-6578

[10] He K M, Zhang X Y, Ren S Q, et al. Deep residual learning for image recognition[C] //Proceedings of the IEEE Conference on Computer Vision and Pattern Recognition. Los Alamitos: IEEE Computer Society Press, 2016: 770-778

[11] Lin T Y, Dollár P, Girshick R, et al. Feature pyramid networks for object detection[C] //Proceedings of the IEEE Conference on Computer Vision and Pattern Recognition. Los Alamitos: IEEE Computer Society Press, 2017: 936-944

[12] Zhang Siyu, Zhang Yi. Small target pedestrian detection based on multi-scale feature fusion[J]. Computer Engineering \& Science, 2019, 41(9): 1627-1634(in Chinese) (张思宇, 张轶. 基于多尺度特征融合的小目标行人检测 [J]. 计算机工程与科学, 2019, 41(9): 1627-1634)

[13] Zhang Dongmin, Jin Guoqing, Dai Feng, et al. Salient object detection based on deep fusion of hand-crafted features[J]. Chinese Journal of Computers, 2019, 42(9): 2076-2086(in Chinese) (张冬明, 靳国庆, 代锋, 等. 基于深度融合的显著性目标检 测算法[J]. 计算机学报, 2019, 42(9): 2076-2086)

[14] Zhan Yi, Li Shengjie, Li Meng. Adaptive neighborhood filtering method of image interpolation[J]. Computer Engineering, 2015, 41(2): 224-227+233(in Chinese)

(詹毅, 李声杰, 李梦. 图像插值的自适应邻域滤波方法[J]. 计算机工程, 2015, 41(2): 224-227+233)

[15] Lin T Y, Maire M, Belongie S, et al. Microsoft COCO: common objects in context[C] //Proceedings of European Conference on Computer Vision. Heidelberg: Springer, 2014: 740-755

[16] Szegedy C, Ioffe S, Vanhoucke V, et al. Inception-v4, inception-ResNet and the impact of residual connections on learning[C] //Proceedings of the 31st AAAI Conference on Artificial Intelligence. Palo Alto: AAAI Press, 2017: 4278-4284

[17] Dai J F, Qi H Z, Xiong Y W, et al. Deformable convolutional networks[C] //Proceedings of the IEEE International Conference on Computer Vision. Los Alamitos: IEEE Computer Society Press, 2017: 764-773

[18] Wang X Y, Yang M, Zhu S H, et al. Regionlets for generic object detection[C] //Proceedings of the IEEE International Conference on Computer Vision. Los Alamitos: IEEE Computer Society Press, 2013: 17-24

[19] He K M, Gkioxari G, Dollár P, et al. Mask R-CNN[C] // Proceedings of the IEEE International Conference on Computer Vision. Los Alamitos: IEEE Computer Society Press, 2017: 2980-2988

[20] Xie S N, Girshick R, DollárP, et al. Aggregated residual transformations for deep neural networks[C] //Proceedings of the IEEE Conference on Computer Vision and Pattern Recognition. Los Alamitos: IEEE Computer Society Press, 2017: 5987-5995

[21] Redmon J, Farhadi A. YOLO9000: better, faster, stronger[C] // Proceedings of the IEEE Conference on Computer Vision and Pattern Recognition. Los Alamitos: IEEE Computer Society Press, 2017: 6517-6525

[22] Fu C Y, Liu W, Ranga A, et al. DSSD: deconvolutional single shotdetector[OL]. [2020-04-08]. https:arxiv.org/abs/1701.06659

[23] Zhang S F, Wen L Y, Bian X, et al. Single-shot refinement neural network for object detection[C] //Proceedings of the IEEE Conference on Computer Vision and Pattern Recognition. Los Alamitos: IEEE Computer Society Press, 2018: 4203-4212 